hep-ph/0403121

\title{
Nucleon contribution to the induced charge of neutrinos in a matter background and a magnetic field
}

\author{
José F. Nieves \\ Laboratory of Theoretical Physics, Department of Physics, P.O. Box 23343, \\ University of Puerto Rico Río Piedras, Puerto Rico 00931-3343
}

(Dated: March 2004)

\begin{abstract}
We study the nucleon contribution to the electromagnetic vertex function of neutrinos that propagate in a matter background in the presence of a magnetic field. Starting from the one-loop expression for the corresponding terms of the vertex function, and taking into account the anomalous magnetic coupling of the nucleons, we calculate the $B$-dependent part of the form factors that determine the induced charge of the neutrino. A formula for the neutrino induced charge is obtained, and it is evaluated for various illustrative situations. The terms due to the nucleons can be important in some cases, depending on the physical conditions of the environment.
\end{abstract}

\section{INTRODUCTION AND SUMMARY}

In the physical contexts in which the effects of an electron background are being considered, in principle a nucleon background is also involved. In some situations, and depending on the effects being studied, the presence of the nucleons is not particularly relevant, in which case they can be thought to form an inert background that makes the medium electrically neutral overall, with no further physical implications. For example, for a photon that propagates through a matter background, the proton contribution to the plasma frequency is insignificant compared to the electron one, since the corresponding terms are proportional to the inverse of the charged particle mass. In most cases, the effects of the nucleons on the photon dispersion relation turn out to be unimportant.

The situation is different when we consider neutrinos instead of photons. When a neutrino propagates in a background of normal matter (electrons and nucleons), the contributions of the various background particle species to the neutrino dispersion relation are comparable, each being proportional to the background particle number density [1]. As is well known, the nucleon contribution is identical for the three neutrino flavors, and it is not relevant in the context of neutrino oscillations in matter that involve only the standard flavor neutrinos, since the oscillation phenomena depends on the difference in the dispersion relations. However, for example, if the so-called sterile neutrinos also take part in the oscillation phenomena, then the nucleon contribution must be included since it is of the same magnitude as the electron contribution.

Besides the dispersion relation, another quantity of interest is the effective electromagnetic coupling of the neutrino in the background medium 2, 3, 4, 5, 6, 6, 8]. That coupling is the basis of some of the neutrino processes that can occur in a medium but not in the vacuum, such as the plasmon decay and the Cherenkov radiation process [1]. In addition, in the presence of a magnetic field, the electromagnetic coupling induces a modification of the neutrino dispersion relation 9 , 10, 11, 12] which is the basis for the asymmetric neutrino emission phenomena that has been considered in various astrophysical contexts 13, 14, 15, 16, 17, 18, 19, 20]. The neutrino electromagnetic interactions also modify the collective properties of a plasma, which can lead to a variety of effects [21, 22, 23, 24, 25].

For calculational purposes, the electromagnetic coupling is parametrized by the form factors of the vertex function 26, 27, 28]. The calculations of the nucleon background contribution to the form factors reveal that it can be of the same magnitude as the one due to the background electrons [29]. Thus, for example, in the context of the asymmetric neutrino emission process already mentioned, the nucleon contribution is unimportant if only the standard flavor neutrinos are considered, but they are significant and must be included if sterile neutrinos are involved.

There has been interest recently in the calculation of the neutrino electromagnetic coupling in the presence of a magnetic field. The calculation in Ref. [30] applies if the particle number densities in the medium are relatively small, such that they can be neglected entirely. A calculation taking into account the electrons in the background was performed in Ref. [31], and it was extended and some aspects were clarified in Ref. [32]. In Ref. [32] the focus was on the form factors of the full vertex function, while Ref. [31] was focused on the particular term of the vertex function that can be identified as the induced neutrino charge. In spite of these differences, the calculations have in common that they neglect the effects of the nucleons in the medium. For some applications, such simplification may be appropriate. However, as we have mentioned above, that it is not always the case and, therefore, the results of the pertinent calculations are needed. Such calculations are the focus of the present work.

Here we consider the effects of the nucleon background on the electromagnetic coupling of the neutrino, in the presence of a magnetic field $B$. In particular, we take into account the anomalous magnetic moment interaction of the nucleons with the magnetic field and, as in Refs. 31] and 32], our goal is to determine those corrections that are linear in $B$. We follow the treatment of the latter reference, adapted to the present situation. An essential ingredient 
in our previous calculation was the linear approximation to the Schwinger formula for the electron propagator in a magnetic field, extended to include the presence of the electron background. Here we derive the corresponding formula for the nucleon propagator, which takes into account the anomalous magnetic coupling of the nucleons. Using that propagator we obtain the expression for the one-loop contribution to the neutrino vertex function. Although the vertex function can be decomposed in terms of a set of tensors as discussed in Ref. [32], here we do not give explicitly the integral formulas for all the associated form factors in the general case, which are cumbersome and not illuminating. For definiteness, we carry out in detail the evaluation of the vertex function in the so-called static and long wavelength limit, which can be interpreted in terms of the neutrino effective charge in the medium. We give the formula for the nucleon contribution to that quantity, in terms of momentum integrals over the nucleon number densities, and in order to establish a point of comparison with the previous calculations we evaluate it explicitly for various illustrative cases. As we have anticipated, the nucleon and the electron contributions can be comparable, depending on the conditions of the environment. Our calculations can be useful for the studies of the effects of the neutrino electromagnetic interactions in various astrophysical environments, which have been considered in the literature.

\section{THE LINEARIZED NUCLEON PROPAGATOR}

The propagator that was used in the calculation of Ref. [32] was obtained by taking the Schwinger formula for the electron propagator in a magnetic field, and expanding it up to linear terms in $B$. We want to obtain the corresponding formula for the nucleon propagator, and we follow closely the notation of that reference. But in order to include the anomalous magnetic moment coupling, here we proceed as follows.

In the presence of the magnetic field, the propagator of each nucleon $f=n, p$ satisfies the equation

$$
\left[i \not \partial-e_{f} \not A-m_{f}-\frac{1}{2} \kappa_{f} \sigma \cdot F\right] S\left(x, x^{\prime}\right)=\delta^{(4)}\left(x-x^{\prime}\right),
$$

where $e_{p}=|e|$ and $e_{n}=0$, and $\kappa_{f}$ is the anomalous magnetic moment, given by

$$
\begin{aligned}
\kappa_{p} & =1.79\left(\frac{|e|}{2 m_{p}}\right), \\
\kappa_{n} & =-1.91\left(\frac{|e|}{2 m_{n}}\right),
\end{aligned}
$$

with $e$ being the electron charge. We have used the notation $\sigma \cdot F=\sigma_{\mu \nu} F^{\mu \nu}$, where $\sigma_{\mu \nu}=(i / 2)\left[\gamma_{\mu}, \gamma_{\nu}\right]$, and $F^{\mu \nu}$ is the electromagnetic tensor for the $B$ field, which we can write in the form

$$
F_{\mu \nu}=i B P_{\mu \nu},
$$

with

$$
P_{\mu \nu}=i \epsilon_{\mu \nu \alpha \beta} b^{\alpha} u^{\beta} .
$$

In an arbitrary frame of reference, the vector $u^{\mu}$ in Eq. (2.4) is the velocity four-vector of the medium. In the frame in which the medium is at rest, which we adopt, $u^{\mu}$ and $b^{\mu}$ take the form

$$
\begin{aligned}
u^{\mu} & =(1, \overrightarrow{0}), \\
b^{\mu} & =(0, \hat{b}),
\end{aligned}
$$

and the magnetic field is given by

$$
\vec{B}=B \hat{b} .
$$

Furthermore, for definiteness, we choose the gauge such that

$$
A_{\mu}=-\frac{1}{2} F_{\mu \nu} x^{\nu} .
$$

The idea now is to write

$$
S\left(x, x^{\prime}\right)=\phi\left(x, x^{\prime}\right) \int \frac{d^{4} p}{(2 \pi)^{4}} e^{-i p \cdot\left(x-x^{\prime}\right)} S_{F}(p),
$$


where

$$
\phi\left(x, x^{\prime}\right)=e^{\frac{i}{2} e_{f} x^{\mu} F_{\mu \nu} x^{\prime \nu}},
$$

and substitute it in Eq. (2.1) to obtain an equation for the momentum space propagator $S_{F}(p)$. Using

$$
i \partial_{\mu} \phi=-\frac{e_{f}}{2} F_{\mu \nu} x^{\prime \nu} \phi
$$

it follows that

$$
\left(i \partial_{\mu}-e_{f} A_{\mu}\right) S\left(x, x^{\prime}\right)=\phi \int \frac{d^{4} p}{(2 \pi)^{4}} e^{-i p \cdot\left(x-x^{\prime}\right)}\left[p_{\mu}-\frac{i e_{f}}{2} F_{\mu \nu} \frac{\partial}{\partial p_{\nu}}\right] S_{F}(p),
$$

and therefore this procedure leads to

$$
\left[\not p-\frac{i e_{f}}{2} F^{\mu \nu} \gamma_{\mu} \frac{\partial}{\partial p^{\nu}}-m_{f}-\frac{1}{2} \kappa_{f} \sigma \cdot F\right] S_{F}(p)=1 .
$$

This equation continues to hold in another gauge, different from Eq. (2.7), as long as $\phi$ is chosen such that is satisfies Eq. (2.10). In general, in another gauge $\phi$ is not be given by Eq. (2.9).

Since we are interested in calculating the effects that are linear in $B$, to solve Eq. (2.12) we substitute

$$
S_{F}(p)=S_{0}+S_{B}
$$

where

$$
S_{0}=\frac{1}{\not p-m_{f}+i \epsilon}
$$

and then solve for $S_{B}$ by iteration. This yields

$$
S_{B}=S_{0}\left(\frac{i e_{f}}{2} F^{\mu \nu} \gamma_{\mu} \frac{\partial}{\partial p^{\nu}}+\frac{1}{2} \kappa_{f} \sigma \cdot F\right) S_{0},
$$

which after using the relation

$$
\frac{1}{2} \sigma \cdot F=B \gamma_{5} \psi \psi
$$

and a little bit of Dirac algebra, can be written in the form

$$
S_{B}=\frac{1}{\left(p^{2}-m_{f}^{2}+i \epsilon\right)^{2}}\left[e_{f} B G+\kappa_{f} B H\right],
$$

where

$$
\begin{aligned}
& G(p)=\gamma_{5}\left[(p \cdot b) \psi-(p \cdot u) \not b+m_{f} \psi \not b\right], \\
& H(p)=\left(\not p+m_{f}\right) \gamma_{5} \psi \not b\left(\not p+m_{f}\right) .
\end{aligned}
$$

The thermal propagator, which incorporates the presence of the nucleons in the background, is given as usual by $[33$.

$$
S_{f}=S_{F}-\left[S_{F}-\bar{S}_{F}\right] \eta_{f}
$$

where

$$
\eta_{f}(p \cdot u)=\theta(p \cdot u) f_{f}(p \cdot u)+\theta(-p \cdot u) f_{\bar{f}}(-p \cdot u),
$$

with

$$
\begin{aligned}
& f_{f}(x)=\frac{1}{e^{\beta\left(x-\mu_{f}\right)}+1}, \\
& f_{\bar{f}}(x)=\frac{1}{e^{\beta\left(x+\mu_{f}\right)}+1} .
\end{aligned}
$$




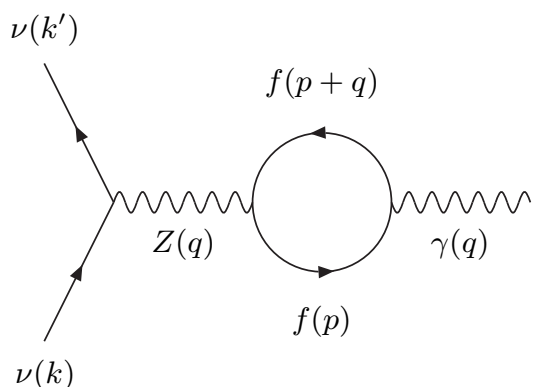

FIG. 1: One-loop diagram for the contribution of a background nucleon $f=n, p$ to the neutrino electromagnetic vertex.

Here $\beta$ stands for the inverse temperature and $\mu_{f}$ for the chemical potential of the nucleon $f$. Using Eq. (2.13), together with (2.17) and (2.18), the complete propagator, linear in $B$, can be expressed as

$$
S_{f}=S_{0}+S_{B}+S_{T}+S_{T B}
$$

where $S_{0}$ and $S_{B}$ are given in Eqs. (2.14) and (2.17), while

$$
\begin{aligned}
i S_{T} & =-2 \pi \delta\left(p^{2}-m_{f}^{2}\right) \eta_{f}\left(\not p+m_{f}\right) \\
i S_{T B} & =2 \pi \delta^{\prime}\left(p^{2}-m_{f}^{2}\right) \eta_{f}\left[e_{f} B G+\kappa_{f} B H\right],
\end{aligned}
$$

with $G$ and $H$ given in Eq. (2.18). The notation $\delta^{\prime}$ denotes the derivative of the delta function with respect to its argument.

As a simple verification of these formulas, we can consider the case of the electron, so that we can set $\kappa_{f}=0$ and $e_{f}=-|e|$ in Eqs. (2.17) and (2.23). In this case the resulting formula for the propagator precisely coincides with the formula that was obtained in Ref. 32] by expanding directly the Schwinger formula, as it should be. We have also used this propagator to compute the nucleon contribution to the neutrino self-energy in a matter background in the presence of a $B$ field, and we have verified that the known result [29] is reproduced.

\section{NUCLEON CONTRIBUTION TO THE VERTEX FUNCTION}

The total background-dependent part of the vertex function for a neutrino of a given flavor $\ell=e, \mu \tau$ will be denoted by $\Gamma_{\lambda}^{\left(\nu_{\ell}\right)}$. Here we denote by $\Gamma_{\lambda}^{(e)}$ the electron contribution, which was calculated in Ref. [32], and write

$$
\Gamma_{\lambda}^{\left(\nu_{\ell}\right)}=\Gamma_{\lambda}^{(e)}+\Gamma_{\lambda}^{(\mathrm{nucl})}
$$

The subject of the present paper is the calculation of the nucleon contribution $\Gamma_{\lambda}^{(\text {nucl) }}$, for which the relevant diagram is the one shown in Fig. 1]

\section{A. Calculation of the vertex function}

For each nucleon in the loop, the propagator is given in Eq. 2.22), while the electromagnetic couplings are given by

$$
L_{\gamma}=-|e| A^{\mu} \bar{p} \gamma_{\mu} p-\frac{\kappa_{p}}{2} \bar{p} \sigma^{\mu \nu} p F_{\mu \nu}-\frac{\kappa_{n}}{2} \bar{n} \sigma^{\mu \nu} n F_{\mu \nu} .
$$

For the neutral-current couplings we write

$$
L_{Z}=-g_{Z} Z^{\mu}\left[\sum_{\ell=e, \mu, \tau} \bar{\nu}_{L \ell} \gamma_{\mu} \nu_{L \ell}+\sum_{f} \bar{f} \gamma_{\mu}\left(a_{f}+b_{f} \gamma_{5}\right) f\right],
$$


where

$$
\begin{aligned}
g_{Z} & =\frac{g}{2 \cos \theta_{W}}, \\
-a_{e}=a_{p} & =\frac{1}{2}-2 \sin ^{2} \theta_{W}, \\
a_{n} & =-\frac{1}{2}, \\
b_{e} & =\frac{1}{2} \\
b_{n}=-b_{p} & =\frac{1}{2} g_{A} .
\end{aligned}
$$

In Eq. (3.4), $g_{A}$ is the normalization constant of the axial-vector current of the nucleon, $g_{A}=1.26$. As depicted in the diagram, we denote by $k$ and $k^{\prime}$ the momentum of the incoming and outgoing neutrino, respectively, and

$$
q=k^{\prime}-k,
$$

is the momentum of the incoming photon.

With these conventions, the diagram in Fig. 1 leads to

$$
\Gamma_{\lambda}^{(\mathrm{nucl})}=-\frac{g^{2}}{4 M_{W}^{2}}\left(T_{\mu \lambda}^{(p)}+T_{\mu \lambda}^{(n)}\right) \gamma^{\mu} L
$$

where $L=\frac{1}{2}\left(1-\gamma_{5}\right)$ as usual, and

$$
T_{\mu \lambda}^{(f)}=i \int \frac{d^{4} p}{(2 \pi)^{4}} \operatorname{Tr}\left[\gamma_{\mu}\left(a_{f}+b_{f} \gamma_{5}\right) S_{f}(p+q) j_{f \lambda}^{(\mathrm{em})} S_{f}(p)\right] .
$$

In Eq. (3.7), $j_{f \lambda}^{(\mathrm{em})}$ is the total electromagnetic current of each nucleon,

$$
\begin{aligned}
& j_{p \lambda}^{(\mathrm{em})}=|e| \gamma_{\lambda}+i \kappa_{p} \sigma_{\lambda \nu} q^{\nu}, \\
& j_{n \lambda}^{(\mathrm{em})}=i \kappa_{n} \sigma_{\lambda \nu} q^{\nu} .
\end{aligned}
$$

When Eq. (2.22) is substituted in Eq. (3.7) several terms are produced. We are interested in the ones that depend on $B$ and the distribution functions which, schematically, involve the products $S_{0} S_{T B}$ or $S_{T} S_{B}$. Denoting the sum of all such terms by $T_{\mu \lambda}^{\prime(f)}$, then

$$
\begin{aligned}
T_{\mu \lambda}^{\prime(f)}= & i \int \frac{d^{4} p}{(2 \pi)^{4}} \operatorname{Tr}\left[\gamma_{\mu}\left(a_{f}+b_{f} \gamma_{5}\right) S_{0}(p+q) j_{f \lambda}^{(\mathrm{em})} S_{T B}(p)\right. \\
& +\gamma_{\mu}\left(a_{f}+b_{f} \gamma_{5}\right) S_{T B}(p+q) j_{f \lambda}^{(\mathrm{em})} S_{0}(p) \\
& +\gamma_{\mu}\left(a_{f}+b_{f} \gamma_{5}\right) S_{B}(p+q) j_{f \lambda}^{(\mathrm{em})} S_{B}(p) \\
& \left.+\gamma_{\mu}\left(a_{f}+b_{f} \gamma_{5}\right) S_{B}(p+q) j_{f \lambda}^{(\mathrm{em})} S_{B}(p)\right]
\end{aligned}
$$

Using the formulas given Eqs. (2.14), (2.17) and (2.23), $T_{\mu \lambda}^{\prime(f)}$ can be written in the form

$$
T_{\mu \lambda}^{\prime(f)}=a_{f} T_{\mu \lambda}^{(f V)}-b_{f} T_{\mu \lambda}^{(f A)},
$$

where

$$
\begin{aligned}
& T_{\mu \lambda}^{(f V)}=-4 B \int \frac{d^{4} p}{(2 \pi)^{3}} \eta_{f}(p . u)\left\{\frac{-L_{\mu \lambda}^{(1)} \delta^{\prime}\left(p^{2}-m_{f}^{2}\right)}{(p+q)^{2}-m_{f}^{2}}+\frac{L_{\mu \lambda}^{(2)} \delta\left(p^{2}-m_{f}^{2}\right)}{\left[(p+q)^{2}-m_{f}^{2}\right]^{2}}-(q \rightarrow-q)\right\}, \\
& T_{\mu \lambda}^{(f A)}=-4 B \int \frac{d^{4} p}{(2 \pi)^{3}} \eta_{f}(p . u)\left\{\frac{-K_{\mu \lambda}^{(1)} \delta^{\prime}\left(p^{2}-m_{f}^{2}\right)}{(p+q)^{2}-m_{f}^{2}}+\frac{K_{\mu \lambda}^{(2)} \delta\left(p^{2}-m_{f}^{2}\right)}{\left[(p+q)^{2}-m_{f}^{2}\right]^{2}}+(q \rightarrow-q)\right\},
\end{aligned}
$$


with the the definitions

$$
\begin{aligned}
4 L_{\mu \lambda}^{(1)}(p, q) & =\operatorname{Tr} \gamma_{\mu}\left(\not p+\not q+m_{f}\right) j_{f \lambda}^{(\mathrm{em})}\left[e_{f} G(p)+\kappa_{f} H(p)\right], \\
4 L_{\mu \lambda}^{(2)}(p, q) & =\operatorname{Tr} \gamma_{\mu}\left[e_{f} G(p)+\kappa_{f} H(p)\right] j_{f \lambda}^{(\mathrm{em})}\left(\not p+\not q+m_{f}\right), \\
4 K_{\mu \lambda}^{(1)}(p, q) & =\operatorname{Tr} \gamma_{5} \gamma_{\mu}\left(\not p+\not q+m_{f}\right) j_{f \lambda}^{(\mathrm{em})}\left[e_{f} G(p)+\kappa_{f} H(p)\right], \\
4 K_{\mu \lambda}^{(2)}(p, q) & =\operatorname{Tr} \gamma_{5} \gamma_{\mu}\left[e_{f} G(p)+\kappa_{f} H(p)\right] j_{f \lambda}^{(\mathrm{em})}\left(\not p+\not q+m_{f}\right) .
\end{aligned}
$$

In arriving at Eq. (3.11) we have applied the usual trick of making the change of variable $p \rightarrow p-q$ in those terms of the integrand that contain the factor $\eta_{f}((p+q) \cdot u)$, and used the relations

$$
\begin{aligned}
L_{\mu \lambda}^{(1)}(p-q, q) & =-L_{\lambda \mu}^{(2)}(p,-q), \\
K_{\mu \lambda}^{(1)}(p-q, q) & =K_{\lambda \mu}^{(2)}(p,-q),
\end{aligned}
$$

and the corresponding ones with the indices 1 and 2 interchanged. The relationships given in Eq. (3.13) can be established by manipulating the order of the factors in the traces defined in Eq. (3.12) with the help of the identity $C^{-1} \gamma_{\mu} C=-\gamma_{\mu}^{T}$, where $C$ is the charge conjugation matrix $\left(C=i \gamma_{2} \gamma_{0}\right.$ in the Dirac representation).

\section{B. Structure of the vertex function}

It follows from inspection of Eq. (3.11) that $T_{\mu \lambda}^{(f V)}$ transforms as a pseudo-tensor under parity. Further, $T_{\mu \lambda}^{(f V)}$ and $T_{\mu \lambda}^{(f A)}$ satisfy the transversality relations

$$
\begin{aligned}
q^{\lambda} T_{\mu \lambda}^{(f V)}=q^{\mu} T_{\mu \lambda}^{(f V)} & =0, \\
q^{\lambda} T_{\mu \lambda}^{(f A)} & =0,
\end{aligned}
$$

which follow from the conservation of the electromagnetic current and the vector neutral-current. We do not show it here, but in fact they can be proven explicitly in the present context by starting from Eq. (3.7) and then using the equation satisfied by the propagator, given in Eq. (2.12).

The most efficient way to calculate $T_{\mu \lambda}^{(f V)}$ and $T_{\mu \lambda}^{(f A)}$ is to decompose them in terms of a complete set of tensors, consistently with the above properties, and then evaluate the corresponding form factors. For this purpose it is useful to introduce the vectors

$$
\begin{aligned}
& \tilde{u}_{\mu}=\tilde{g}_{\mu \nu} u^{\nu}, \\
& \tilde{b}_{\mu}=\tilde{g}_{\mu \nu} b^{\nu}-\frac{q \cdot u}{q^{2}}\left(q \cdot u b_{\mu}-q \cdot b u_{\mu}\right), \\
& \tilde{t}_{\mu}=\epsilon_{\mu \alpha \beta \gamma} u^{\alpha} b^{\beta} q^{\gamma},
\end{aligned}
$$

where

$$
\tilde{g}_{\mu \nu} \equiv g_{\mu \nu}-\frac{q_{\mu} q_{\nu}}{q^{2}}
$$

which are orthogonal to $q^{\mu}$. Following Ref. [32], we can decompose $T_{\mu \lambda}^{(f V)}$ and $T_{\mu \lambda}^{(f A)}$ in the form

$$
\begin{aligned}
& T_{\mu \lambda}^{(f V)}=\tilde{T}_{1}^{(f V)} \tilde{P}_{1 \mu \lambda}+\tilde{T}_{2}^{(f V)} \tilde{P}_{2 \mu \lambda}+\tilde{T}_{S}^{(f V)} S_{2 \mu \lambda}, \\
& T_{\mu \lambda}^{(f A)}=T_{L}^{(f A)} Q_{\mu \lambda}+T_{T}^{(f A)}\left(\tilde{g}_{\mu \lambda}-Q_{\mu \lambda}\right)+T_{A}^{(f A)} A_{3 \mu \lambda}+T_{S}^{(f A)} S_{3 \mu \lambda}+T_{u}^{(f A)} q_{\mu} \tilde{u}_{\lambda}+T_{b}^{(f A)} q_{\mu} \tilde{b}_{\lambda},
\end{aligned}
$$

where the various tensors that appear here are defined as follows,

$$
\begin{aligned}
Q_{\mu \lambda} & =\frac{\tilde{u}_{\mu} \tilde{u}_{\lambda}}{\tilde{u}^{2}}, \\
S_{2 \mu \lambda} & =\tilde{u}_{\mu} \tilde{t}_{\lambda}+(\mu \leftrightarrow \lambda), \\
S_{3 \mu \lambda} & =\tilde{u}_{\mu} \tilde{b}_{\lambda}+(\mu \leftrightarrow \lambda), \\
A_{3 \mu \lambda} & =\tilde{u}_{\mu} \tilde{b}_{\lambda}-(\mu \leftrightarrow \lambda), \\
\tilde{P}_{1 \mu \lambda} & =i \epsilon_{\mu \lambda \alpha \beta} \tilde{u}^{\alpha} q^{\beta}, \\
\tilde{P}_{2 \mu \lambda} & =i \epsilon_{\mu \lambda \alpha \beta} \tilde{b}^{\alpha} q^{\beta} .
\end{aligned}
$$


In Eq. (3.17) we have included a term proportional to $S_{2 \mu \lambda}$ because, in contrast to the case of the electron background contribution, $T_{\mu \lambda}^{(f V)}$ is not antisymmetric.

The form factors are functions of the three scalar variables $\omega, Q_{\|}$and $Q_{\perp}$ which are defined by

$$
\begin{aligned}
\omega & =q \cdot u, \\
Q_{\|} & =-q \cdot b, \\
Q_{\perp} & =\sqrt{Q^{2}-Q_{\|}^{2}},
\end{aligned}
$$

where

$$
Q^{2}=|\vec{Q}|^{2}=\omega^{2}-q^{2}
$$

The integral expressions for the form factors are obtained by contracting both sides of Eqs. (3.17) and (3.18) with the various tensors that appear in those expansions. For example, the form factors $T_{L}^{(f A)}$ and $T_{u}^{(f A)}$, which enter in the definition of the neutrino induced charge, are given by

$$
\begin{aligned}
& T_{L}^{(f A)}=-\frac{4 B}{\tilde{u}^{2}} \int \frac{d^{4} p}{(2 \pi)^{3}} \eta_{f}(p . u)\left\{\frac{-\tilde{u}^{\mu} \tilde{u}^{\lambda} K_{\mu \lambda}^{(1)} \delta^{\prime}\left(p^{2}-m_{f}^{2}\right)}{(p+q)^{2}-m_{f}^{2}}+\frac{\tilde{u}^{\mu} \tilde{u}^{\lambda} K_{\mu \lambda}^{(2)} \delta\left(p^{2}-m_{f}^{2}\right)}{\left[(p+q)^{2}-m_{f}^{2}\right]^{2}}+(q \rightarrow-q)\right\}, \\
& T_{u}^{(f A)}=-\frac{4 B}{q^{2} \tilde{u}^{2}} \int \frac{d^{4} p}{(2 \pi)^{3}} \eta_{f}(p . u)\left\{\frac{-q^{\mu} \tilde{u}^{\lambda} K_{\mu \lambda}^{(1)} \delta^{\prime}\left(p^{2}-m_{f}^{2}\right)}{(p+q)^{2}-m_{f}^{2}}+\frac{q^{\mu} \tilde{u}^{\lambda} K_{\mu \lambda}^{(2)} \delta\left(p^{2}-m_{f}^{2}\right)}{\left[(p+q)^{2}-m_{f}^{2}\right]^{2}}-(q \rightarrow-q)\right\} .
\end{aligned}
$$

In the general case, the formulas so obtained are cumbersome and not very illuminating, and for this reason we do not proceed any further along this direction. Instead, we consider specifically the calculation of the form factors in the static and long wavelength limit, which are related to the effective neutrino charge in the medium. In this way we are able to obtain approximate formulas that can be used in practical situations, and it will allow us to compare the importance of the nucleon background contribution, relative to the electron background.

\section{NEUTRINO EFFECTIVE CHARGE}

We consider the calculation of the induced charge of the neutrino, and specifically the parameter that was denoted by $e_{\nu_{\ell}}^{\|}$in Ref. [32]. We denote by $\Gamma_{\mu}\left(\omega, Q_{\perp}, Q_{\|}\right)$the neutrino vertex function evaluated for an arbitrary value of $q$, and use a similar notation for the form factors as well. With this notation, $e_{\nu_{\ell}}^{\|}$is defined by

$$
e_{\nu_{\ell}}^{\|}=\frac{1}{2 E_{\nu}} \operatorname{Tr} L \not k \Gamma_{0}\left(0,0, Q_{\|} \rightarrow 0\right),
$$

where $k^{\mu}=\left(E_{\nu}, \vec{k}\right)$ is the neutrino momentum vector.

\section{A. Nucleon contribution}

Using the same notation for the nucleon contribution, the quantity that we wish to calculate is

$$
e_{\text {nucl }}^{\|}=\frac{1}{2 E_{\nu}} \operatorname{Tr} L \not k \Gamma_{0}^{(\mathrm{nucl})}\left(0,0, Q_{\|} \rightarrow 0\right) .
$$

In practice, we can evaluate the vertex function in this limit by first setting

$$
q^{\mu}=Q_{\|} b^{\mu}
$$

and then taking $Q_{\|} \rightarrow$ afterwards. From the definition of the various tensors given in Eq. (3.19), it then follows that

$$
\Gamma_{0}^{(\mathrm{nucl})}\left(0,0, Q_{\|}\right)=\frac{g^{2}}{4 M_{W}^{2}} \sum_{f=n, p} b_{f}\left\{T_{L}^{(f A)} \gamma_{0}-Q_{\|} T_{u}^{(f A)} \vec{\gamma} \cdot \hat{b}\right\} L,
$$


with the form factors evaluated at $\omega=0$ and $Q_{\perp}=0$. Therefore, to evaluate them, we can put $\tilde{u}_{\mu} \rightarrow u_{\mu}$ in Eq. (3.22). We now use a property of the traces that are defined in Eq. (3.12), which we can state precisely by defining

$$
K_{L}^{(i)}(\vec{p}, \vec{q}) \equiv u^{\mu} u^{\lambda} K_{\mu \lambda}^{(i)} \quad(i=1,2)
$$

where we have indicated explicitly the dependence on the spatial components of the momentum vectors. Then, with $q^{\mu}$ as given in Eq. (4.3), it follows from the trace formula in Eq. (3.12) that

$$
K_{L}^{(i)}(-\vec{p},-\vec{q})=-K_{L}^{(i)}(\vec{p}, \vec{q})
$$

Using this property, and the fact that the distribution functions are isotropic in $\vec{p}$, Eq. (3.22) yields

$$
T_{L}^{(f A)}\left(0,0, Q_{\|}\right)=0
$$

Thus, we need to evaluate only $T_{u}^{(f A)}$, which we write in the form

$$
T_{u}^{(f A)}=-\frac{4 B}{q^{2} \tilde{u}^{2}}\left[I_{1}+I_{2}-(q \rightarrow-q)\right],
$$

where

$$
\begin{aligned}
I_{1} & =(-1) \int \frac{d^{4} p}{(2 \pi)^{3}} \eta_{f}(p . u) \frac{F_{1}(p, q) \delta^{\prime}\left(p^{2}-m_{f}^{2}\right)}{(p+q)^{2}-m_{f}^{2}}, \\
I_{2} & =\int \frac{d^{4} p}{(2 \pi)^{3}} \eta_{f}(p . u) \frac{F_{2}(p, q) \delta\left(p^{2}-m_{f}^{2}\right)}{\left[(p+q)^{2}-m_{f}^{2}\right]^{2}},
\end{aligned}
$$

with

$$
F_{i}(p, q) \equiv q^{\mu} \tilde{u}^{\lambda} K_{\mu \lambda}^{(i)} \quad(i=1,2)
$$

In order to calculate he traces that are involved in Eq. (4.10), it is useful to note that if we calculate

$$
F_{1}=\frac{1}{4} q^{\mu} \tilde{u}^{\lambda} \operatorname{Tr} \gamma_{5} \gamma_{\mu}\left(\not p^{\prime}+m_{f}\right) j_{f \lambda}^{(\mathrm{em})}\left[e_{f} G(p)+\kappa_{f} H(p)\right]
$$

where we have put $p^{\prime}=p+q$, then the result for $F_{2}$ is obtained by making the substitution $p^{\prime} \leftrightarrow-p$. After some tedious, but straightforward Dirac algebra, in this way we obtain, for $q$ as given in Eq. (4.3),

$$
\begin{aligned}
& F_{1}=e_{f}^{2} A+e_{f} \kappa_{f} B_{1}+\kappa_{f}^{2} C_{2}, \\
& F_{2}=e_{f}^{2} A+e_{f} \kappa_{f} B_{2}+\kappa_{f}^{2} C_{2},
\end{aligned}
$$

where

$$
\begin{aligned}
A & =-Q_{\|}\left(p^{02}+P_{\|}^{2}+m_{f}^{2}+Q_{\|} P_{\|}\right) \\
B_{2} & =m_{f} Q_{\|}\left(p^{2}-m_{f}^{2}\right)-2 m_{f} Q_{\|}\left(2 p^{02}-Q_{\|}^{2}\right) \\
B_{1} & =B_{2}-m_{f} Q_{\|}^{2}\left(2 P_{\|}+Q_{\|}\right) \\
C_{1} & =Q_{\|}^{2}\left[-\left(3 P_{\|}+Q_{\|}\right)\left(p^{2}-m_{f}^{2}\right)+2\left(2 P_{\|}+Q_{\|}\right)\left(p^{02}-P_{\|}^{2}\right)-4 m_{f}^{2} P_{\|}\right], \\
C_{2} & =-Q_{\|}^{2}\left[-\left(3 P_{\|}+2 Q_{\|}\right)\left(p^{2}-m_{f}^{2}\right)+\left(2 P_{\|}+Q_{\|}\right)\left(2 p^{02}-P_{\|}\left(2 P_{\|}+Q_{\|}\right)\right)-4 m_{f}^{2}\left(P_{\|}+Q_{\|}\right)\right],
\end{aligned}
$$

with

$$
P_{\|}=\vec{p} \cdot \hat{b}
$$

To calculate $T_{u}^{(f A)}\left(0,0, Q_{\|}\right)$we use the long wavelength limit expressions for $I_{1,2}$ that were derived in Ref. [32], which are useful for computing the integrals in the limit that we need. Those expressions for $I_{1,2}$ are obtained by using the auxiliary formula

$$
\int \frac{d^{3} p}{(2 \pi)^{3}} \frac{\mathcal{F}(p)}{\left[q^{2}+\lambda 2 p \cdot q\right]^{n}}=\lambda^{n} \int \frac{d^{3} p}{(2 \pi)^{3}} \frac{\left(\mathcal{F}-\lambda \frac{\vec{Q}}{2} \cdot \frac{d \mathcal{F}}{d \vec{p}}-\lambda \frac{n \omega}{2 E} \mathcal{F}\right)}{[2 E \omega-2 \vec{p} \cdot \vec{Q}]^{n}}
$$


where $\lambda= \pm 1$, and they are valid for $q \ll\langle\mathcal{E}\rangle$, where $\langle\mathcal{E}\rangle$ denotes a typical average energy of the particles in the background. Thus [34],

$$
\begin{aligned}
& I_{1}=\int \frac{d^{3} p}{(2 \pi)^{3}}\left\{\frac{F_{1}^{\prime-}-\frac{\vec{Q}}{2} \cdot \frac{d F_{1}^{\prime+}}{d \vec{p}}-\frac{\omega}{2 E} F_{1}^{\prime+}}{2 E \omega-2 \vec{p} \cdot \vec{Q}}-\frac{F_{1}^{+}-\frac{\vec{Q}}{2} \cdot \frac{d F_{1}^{-}}{d \overrightarrow{\vec{p}}}}{[2 E \omega-2 \vec{p} \cdot \vec{Q}]^{2}}\right\}, \\
& I_{2}=\int \frac{d^{3} p}{(2 \pi)^{3}} \frac{F_{2}^{+}-\frac{\vec{Q}}{2} \cdot \frac{d F_{2}^{-}}{d \vec{p}}-\frac{\omega}{E} F_{2}^{-}}{[2 E \omega-2 \vec{p} \cdot \vec{Q}]^{2}}
\end{aligned}
$$

where

$$
\begin{aligned}
F_{1}^{ \pm}(p, q) & =\left.\left[\frac{f_{e}\left(p^{0}\right) F_{1}(p, q) \pm f_{\bar{e}}\left(p^{0}\right) F_{1}(-p, q)}{2 p^{0}}\right]\right|_{p^{0}=E(p)} \\
F_{2}^{ \pm}(p, q) & =\left.\left[\frac{f_{e}\left(p^{0}\right) F_{2}(p, q) \pm f_{\bar{e}}\left(p^{0}\right) F_{2}(-p, q)}{2 p^{0}}\right]\right|_{p^{0}=E(|\vec{p}|)} \\
F_{1}^{\prime \pm}(p, q) & =\left.\left\{\frac{1}{2 p^{0}} \frac{\partial}{\partial p^{0}}\left[\frac{f_{e}\left(p^{0}\right) F_{1}(p, q) \pm f_{\bar{e}}\left(p^{0}\right) F_{1}(-p, q)}{2 p^{0}}\right]\right\}\right|_{p^{0}=E(|\vec{p}|)}
\end{aligned}
$$

In Eq. (4.16) the symbol $\frac{d}{d \vec{p}}$ stands for the total momentum derivative,

$$
\frac{d}{d \vec{p}}=\frac{\partial}{\partial \vec{p}}+\frac{\vec{p}}{E} \frac{\partial}{\partial E}
$$

The final step consists in putting $\omega=0$ and $\vec{Q}=Q_{\|} \hat{b}$ in Eq. (4.16) and using Eqs. (4.12) and (4.13) to determine the various terms in the integrands for $I_{1,2}$. The details of this calculation are similar to the corresponding ones for the case of the electron background, some of which were given in Ref. [32], and which we omit here. In this way we arrive at

$$
I_{1}+I_{2}-(q \rightarrow-q)=-Q_{\|}\left[e_{f}^{2} t_{\|}^{(f)}+e_{f} \kappa_{f}\left(2 m_{f} t_{\|}^{(f)}\right)+\kappa_{f}^{2} s_{\|}^{(f)}\right]+O\left(Q_{\|}^{3}\right),
$$

where we define, for any fermion $f$,

$$
\begin{aligned}
t_{\|}^{(f)} & =-\frac{1}{2} \int_{0}^{\infty} \frac{d p}{(2 \pi)^{2}} \frac{\partial}{\partial E}\left[f_{f}(E)+f_{\bar{f}}(E)\right], \\
s_{\|}^{(f)} & =\int \frac{d^{3} p}{(2 \pi)^{3}} \frac{f_{f}(E)+f_{\bar{f}}(E)}{E} .
\end{aligned}
$$

Therefore, from Eq. (4.8),

$$
T^{(f A)}\left(0,0, Q_{\|} \rightarrow 0\right)=-\frac{4 B}{Q_{\|}}\left[e_{f}\left(e_{f}+2 m_{f} \kappa_{f}\right) t_{\|}^{(f)}+\kappa_{f}^{2} s_{\|}^{(f)}\right],
$$

and finally, from Eqs. (4.2) and (4.4), and using the results given in Eqs. (4.7) and (4.22), we obtain

$$
e_{\text {nucl }}^{\|}=\frac{g^{2}}{M_{W}^{2}}(\vec{B} \cdot \hat{k})\left[b_{n} \kappa_{n}^{2} s_{\|}^{(n)}+b_{p}\left(|e|^{2}+2 m_{f}|e| \kappa_{p}\right) t_{\|}^{(p)}+b_{p} \kappa_{p}^{2} s_{\|}^{(p)}\right] .
$$

\section{B. Discussion}

In the environments of physical interest the nucleons are non-relativistic. Therefore, in Eq. (4.21) we can effectively replace $E \rightarrow m_{f}$ in the denominator, so that for the nucleons

$$
s_{\|}^{(f)}=\frac{n_{f}}{2 m_{f}},
$$


where $n_{f}$ is the total number density of the neutrons or protons. Using the result obtained in Ref. [32] for the electron background term, the total matter contribution to the $B$-dependent part the induced neutrino charge is then

$$
e_{\nu_{\ell}}^{\|}=\frac{g^{2}}{M_{W}^{2}}(\vec{B} \cdot \hat{k})\left[-e^{2} \chi_{A}^{(\ell)} t_{\|}^{(e)}+\frac{1}{2 m_{n}} b_{n} \kappa_{n}^{2} n_{n}+\frac{1}{2 m_{p}} b_{p} \kappa_{p}^{2} n_{p}+b_{p}\left(|e|^{2}+2 m_{p}|e| \kappa_{p}\right) t_{\|}^{(p)}\right]
$$

with $t_{\|}^{(e, p)}$ as defined in Eq. (4.20), and

$$
\chi_{A}^{(\ell)}=\delta_{\ell, e}-b_{e}
$$

The additional term that is present for the electron neutrino $(\ell=e)$ is due to the charged-current interaction, which is absent for $\nu_{\mu, \tau}$.

The evaluation of $t_{\|}^{(e, p)}$ requires more care, and we must distinguish various cases. For a relativistic gas, putting $E \simeq p$ in Eq. (4.20) yields

$$
t_{\|}^{(f)}=\frac{1}{8 \pi^{2}} \quad \text { (relativistic) } .
$$

For a non-relativistic (NR) gas, we distinguish two cases. If the gas can be treated classically, then using the relation

$$
\frac{\partial f}{\partial E}=-\beta f
$$

yields

$$
t_{\|}^{(f)}=\frac{n_{f} \beta^{2}}{8 m_{f}} \quad(\mathrm{NR} \text { classical }) .
$$

On the other hand, if the gas is degenerate, we obtain the approximate formula

$$
t_{\|}^{(f)}=\frac{m_{f}}{8 \pi^{2} p_{F}}\left[1+\frac{\pi^{2}}{8} \frac{m_{f}^{2}}{\beta^{2} p_{F}^{4}}\right] \quad \text { (NR degenerate) }
$$

where $p_{F}$ is the Fermi momentum, for

$$
p_{F} \gg \sqrt{m_{f} / \beta} \text {. }
$$

The derivation of Eq. (4.30) is sketched in the Appendix.

The relative importance of the various terms in Eq. (4.25) depends on the value of $t_{\|}^{(e)}$ and $t_{\|}^{(p)}$ which in turn depend on the conditions of the environment. The above approximate formulas are useful in this respect. For example, suppose that the conditions are such that both the electron and proton gases can be treated classically. Then for the protons we use Eq. (4.29). If also the electrons are non-relativistic then we use the same formula for them and, remembering that the charge neutrality of the medium requires that $n_{p}=n_{e}$, it follows that

$$
\frac{t_{\|}^{(p)}}{t_{\|}^{(e)}}=\frac{m_{e}}{m_{p}} \text {. }
$$

On the other hand, if the electrons are relativistic, using Eq. (4.27) for them yields instead

$$
\frac{t_{\|}^{(p)}}{t_{\|}^{(e)}} \sim \frac{T}{m_{p}} .
$$

In either case, we can neglect the $t_{\|}^{(p)}$ term in Eq. (4.25).

In contrast, consider the case in which the gases are degenerate, and for definiteness suppose that the electrons are relativistic. Then using Eq. (4.30) for the protons and Eq. (4.27) for the electrons, it follows that $t_{\|}^{(p)}$ is larger than $t_{\|}^{(e)}$ by a factor $m_{p} / p_{F p}$. Furthermore, the neutron term in Eq. (4.25), as well as the proton term that is proportional 
to $n_{p}$, are of order $n_{n} / m_{n}^{3} \sim p_{F n}^{3} /\left(3 \pi^{2} m_{n}^{3}\right)$ which, for $p_{F n} / m_{n} \sim 1 / 3$, is about a factor of 10 smaller than the electron term $t_{\|}^{(e)}=1 / 8 \pi^{2}$. Therefore, in this case the proton term may be the dominant one in Eq. (4.25).

In any application to the calculation of neutrino processes rates, besides the $B$-dependent coupling just determined, the amplitude contains a $B$-independent term. To have an idea of their relative magnitude we can compare the value of $e_{\nu}^{\|}$with the analogous quantity $e_{\nu}^{(0)}$ calculated in the absence of the $B$ field. From Ref. [5], with a slight change of notation,

$$
e_{\nu}^{(0)}=-\frac{g^{2} \chi_{V}^{(\ell)}}{4 M_{W}^{2}} \frac{1}{|e| r_{D}^{2}}
$$

where $\chi_{V}^{(\ell)}=2 \sim^{2} \theta_{W}-\frac{1}{2}+\delta_{\ell, e}$, and the parameter $r_{D}$ is the Debye screening length, which is given in terms of the longitudinal component of the photon self-energy $\pi_{L}(\omega, Q)$ by

$$
r_{D}^{-2}=\pi_{L}(0, Q \rightarrow 0)
$$

Considering again the case in which the gases are degenerate, with the electrons being relativistic, then we can take 35$]$

$$
r_{D}^{-2}=\frac{e^{2} m_{p} p_{F p}}{\pi^{2}}
$$

neglecting the electron contribution, which is smaller by a factor of $p_{F p} / m_{p}$. Writing

$$
\frac{e_{\nu_{\ell}}^{\|}}{e_{\nu_{\ell}}^{(0)}}=R_{n}+R_{P}+R_{e},
$$

the relative size of the various contributions can be estimated using Eqs. (4.25) and (4.34). For example, for the protons we find

$$
R_{p} \sim \frac{m_{e}^{2} B}{p_{F e}^{2} B_{c}},
$$

where we have used $p_{F P}=p_{F e}$ and $B_{c}=m_{e}^{2} /|e|=4 \times 10^{13} G$, while for the electrons it is a factor of $p_{F e} / m_{p}$ smaller, and it is even smaller for the neutrons as we mentioned above. Since our calculations are based on a weak-field approximation, the implicit assumption is that the magnetic contribution $e_{\nu_{\ell}}^{\|}$is smaller than the zeroth order term

$e_{\nu_{\ell}}^{(0)}$. Nevertheless, it is conceivable that there are physical systems for which $e_{\nu_{\ell}}^{\|}$is not negligible, which can produce observable effects due to its anisotropic nature. The above discussion shows that, in those situations, the nucleon couplings to the $B$ field must be taken into account.

\section{CONCLUSIONS}

A neutrino that propagates through a matter medium acquires an induced electric charge due to its interactions with the matter particles. We have considered the $B$-dependent part of the neutrino electromagnetic vertex, and more specifically the neutrino induced charge, in a background of electrons and nucleons in the presence of a magnetic field. We have extended the previous calculations, which considered only the electrons in the background, to take into account the nucleons and, in particular, to include the effects of their anomalous magnetic coupling. The importance of the contribution due to the nucleons, relative to the electron contribution, depends on the conditions of the environment considered. For example, we indicated that in some situations the term due to the protons is not significant, but there are cases in which the terms due to the nucleons are the most important ones.

Besides the term that we have calculated, which is linear in $B$, the induced charge contains a term that is independent of $B$. The distinctive feature of the $B$-dependent term is that it is not isotropic in the neutrino momentum; it has a different value for neutrinos going in different directions, relative to $\vec{B}$. This could produce observable effects in the context of a variety of physical problems that have been considered in the literature, such as those related to the influence of the neutrino electromagnetic interactions on the collective properties of a plasma. Our results can be useful in those contexts, and this work paves the way for considering and studying those effects in more detail.

\section{Acknowledgments}

This material is based upon work supported by the US National Science Foundation under Grant No. 0139538. 


\section{APPENDIX A: PROOF OF EQ. (4.30)}

Naively, in the completely degenerate limit we can put

$$
f_{f}=\Theta\left(E_{F}-E\right)
$$

in Eq. 4.20) and, assuming that $f_{\bar{f}} \simeq 0$, obtain

$$
t_{\|}^{(f)}=\frac{E_{F}}{8 \pi^{2} p_{F}} .
$$

This agrees with Eq. (4.27) in the relativistic limit, and with the leading term of Eq. (4.30) in the non-relativistic one, but this procedure leaves unanswered the question of the range of validity of this approximation. For that, we consider in more detail the integral involving $f_{f}$ in Eq. (4.20). Similar considerations apply to the other integral, involving the anti-particle distribution, with obvious modifications.

Defining

$$
\begin{aligned}
\epsilon & =E-m_{f}, \\
\mu^{\prime} & =\mu-m_{f},
\end{aligned}
$$

we write

$$
\begin{aligned}
-\int_{0}^{\infty} d p \frac{\partial}{\partial E} \frac{1}{e^{\beta(E-\mu)}+1} & =-\int_{0}^{\infty} d p \frac{\partial}{\partial \epsilon} \frac{1}{e^{\beta\left(\epsilon-\mu^{\prime}\right)}+1} \\
& =\frac{\partial}{\partial \mu^{\prime}} I
\end{aligned}
$$

where

$$
I=\int_{0}^{\infty} d p \frac{1}{e^{\beta\left(\epsilon-\mu^{\prime}\right)}+1}
$$

and changing the integration variable,

$$
I=\int_{0}^{\infty} d \epsilon \frac{g(\epsilon)}{e^{\beta\left(\epsilon-\mu^{\prime}\right)}+1}
$$

with

$$
g(\epsilon)=\frac{E}{p}=\frac{\epsilon+m_{f}}{\left[\left(\epsilon+m_{f}\right)^{2}-m_{f}^{2}\right]^{1 / 2}} .
$$

Letting

$$
z=\beta\left(\epsilon-\mu^{\prime}\right)
$$

$I$ can be written in the form

$$
I=\frac{1}{\beta} \int_{-\beta \mu^{\prime}}^{0} d z g\left(\mu^{\prime}+\frac{z}{\beta}\right) f(z)+\frac{1}{\beta} \int_{0}^{\infty} d z g\left(\mu^{\prime}+\frac{z}{\beta}\right) f(z)
$$

where we have defined

$$
f(z)=\frac{1}{e^{z}+1}
$$

In the first integral in Eq. (A9) we change $z \rightarrow-z$ and use $f(-z)=1-f(z)$, which yields

$$
I=\int_{0}^{\mu^{\prime}} d \epsilon g(\epsilon)-\frac{1}{\beta} \int_{0}^{\beta \mu^{\prime}} d z g\left(\mu^{\prime}-\frac{z}{\beta}\right) f(z)+\frac{1}{\beta} \int_{0}^{\infty} d z g\left(\mu^{\prime}+\frac{z}{\beta}\right) f(z) .
$$


Up to this point this expression is exact. The approximation now consists in replacing the upper limit integration in the second integral by infinity, and it is valid for

$$
\beta \mu^{\prime} \gg 1
$$

This yields the final formula

$$
\frac{\partial}{\partial \mu^{\prime}} I=g\left(\mu^{\prime}\right)+\frac{1}{\beta} \int_{0}^{\infty} d z \frac{g^{\prime}\left(\mu^{\prime}+\frac{z}{\beta}\right)-g^{\prime}\left(\mu^{\prime}-\frac{z}{\beta}\right)}{e^{z}+1}
$$

which can be evaluated explicitly by expanding $g(z)$ in a Taylor series in $z$ and integrating term by term.

Apart from the condition given in Eq. A12, there are no further restrictions, so that this formula can be applied regardless of whether the gas is relativistic or non-relativistic. Thus, for example, in the non-relativistic limit, $g(\epsilon) \approx\left(m_{f} / 2 \epsilon\right)^{1 / 2}$, and from Eq. A13

$$
\frac{\partial}{\partial \mu^{\prime}} I=\sqrt{\frac{m_{f}}{2 \mu^{\prime}}}\left[1+\frac{\pi^{2}}{8 \beta^{2} \mu^{\prime 2}}\right] .
$$

Using this result in Eq. A4, and substituting $\mu^{\prime}=p_{F}^{2} / 2 m_{f}$, leads to the formula quoted in Eq. (4.30).

[1] G. G. Raffelt, Stars as Laboratory for Fundamental Physics (University of Chicago Press, 1996), p. 241, and references therein.

[2] V. B. Semikoz, Physica 142A, 157 (1987).

[3] J. F. Nieves and P. B. Pal, Phys. Rev. D 40, 1693 (1989).

[4] V. N. Oravesky and V. B. Semikoz, Sov. J. Nucl. Phys. 42, 446 (1985).

[5] J. F. Nieves and P. B. Pal, Phys. Rev. D 49, 1398 (1994).

[6] V. N. Oravesky and V. B. Semikoz, Physica 142A, 135 (1987).

[7] V. B. Semikoz, Sov. J. Nucl. Phys. 46, 946 (1987).

[8] R. F. Sawyer, Phys. Rev. D 46, 1180 (1992).

[9] J. C. D'Olivo, J. F. Nieves, and P. B. Pal, Phys. Rev. D 40, 3679 (1989).

[10] A. Erdas, C. W. Kim, and T. H. Lee, Phys. Rev. D 58, 08516 (1998).

[11] S. Esposito and G. Capone, Z. Phys. C 70, 55 (1996).

[12] P. Elmfors, D. Grasso, and G. Raffelt, Nucl. Phys. B 479, 3 (1996).

[13] A. Kusenko and G. Segré, Phys. Rev. Lett. 77, 4872 (1996).

[14] A. Kusenko and G. Segré, Phys. Lett. B 396, 197 (1997).

[15] A. Kusenko and G. Segré, Phys. Rev. D 59, 061302 (1999).

[16] A. Kusenko and G. Segré, Phys. Rev. Lett. 79, 2751 (1997).

[17] Y. Z. Qian, Phys. Rev. Lett. 79, 2750 (1997).

[18] V. B. Semikoz and J. W. F. Valle, Nucl. Phys. B 425, 65 (1994).

[19] V. B. Semikoz and J. W. F. Valle, Nucl. Phys. B 485, 585 (1997).

[20] H. Nunokawa, V. B. Semikoz, A. Y. Smirnov, and J. W. F. Valle, Nucl. Phys. B 501, 17 (1997).

[21] L. O. Silva, R. Bingham, J. M. Dawson, J. T. Mendoz̧a, and P. K. Shukla, Phys. Rev. Lett. 83, 2703 (1999).

[22] L. Bento, Phys. Rev. D 61, 013004 (2000).

[23] J. F. Nieves, Phys. Rev. D 61, 113008 (2000).

[24] S. Mohanty, J. F. Nieves, and P. B. Pal, Phys. Rev. D 58, 093007 (1998).

[25] V. B. Semikoz and D. D. Sokoloff, astro-ph/0312567 (2003).

[26] J. C. D'Olivo and J. F. Nieves, Phys. Lett. B 383, 87 (1996).

[27] T. Altherr and P. Salati, Nucl. Phys. B 421, 662 (1994).

[28] V. B. Semikoz and Y. A. Smorodinsky, Sov. Phys. JETP 68, 20 (1989).

[29] J. C. D'Olivo and J. F. Nieves, Phys. Rev. D 56, 5898 (1997).

[30] A. N. Ioannisian and G. G. Raffelt, Phys. Rev. D 55, 7038 (1997).

[31] K. Bhattacharya, A. Ganguly, and S. Konar, Phys. Rev. D 65, 013007 (2001).

[32] J. F. Nieves, Phys. Rev. D 68, 113003 (2003).

[33] See, e.g., Ref. [12].

[34] We take the opportunity to point out that in Ref. [32], Eq. (5.1) and the second formula in Eq. (5.6) are not written correctly. The correct formulas are given here in Eqs. [4.15] and [4.16), respectively. In the calculations of Ref. 32], the correct formulas were used.

[35] See, e.g., Ref. [1], p. 220. 